\title{
Reagents
}

Formic acid, HPLC grade acetonitrile and methanol were purchased from Thermo Fisher (Thermo Fisher Scientific Inc, USA). In total, 30 Bile acids and 7 deuterium-labeled internal standards (ISs) (see Table 1) were purchased from Steraloids Inc. (Newport, Rhode Island, United States).

\section{Sample preparation}

Intestinal contents $(\sim 10 \mathrm{mg})$ were added to $980 \mu \mathrm{L}$ of extraction solvent (methanol/water (v:v) $=2: 1,0.005 \% \mathrm{HCOOH})$ and $20 \mu \mathrm{L}$ of internal standard and followed with rapid freeze-thaw cycles in liquid nitrogen three times, followed by homogenization using tissulyser at $50 \mathrm{~Hz}$ for $90 \mathrm{~s}$. The extracted supernatant was obtained by centrifugation at $12000 \mathrm{rpm}, 4^{\circ} \mathrm{C}$ for $10 \mathrm{~min}$. Finally, samples were filtered using PTFE filters (Jin Teng, $\varnothing 13 \mathrm{~mm}, 0.22 \mu \mathrm{m}$, Tianjin, China) before UPLCMS/MS analysis. The moisture content is measured separately for each sample.

\section{Calibration curves}

Calibration curve linearity was evaluated by assessing the correlation coefficient $\left(\mathrm{R}^{2}\right)$ of three freshly prepared 12-point calibration curves (see Table 2). Standard curves were constructed by least-squares linear regression analysis using the peak area ratio of a given eicosanoid over its reference IS against the nominal concentration of the calibrator.

The linearity of each bile acid calibration curve was evaluated by correlation coefficient $\left(\mathrm{R}^{2}\right)$, which was generated by least-squares linear regression analysis using the MRM peak area of each bile acid over MRM peak area of the internal standards. Notably, since some of bile acids internal standards were not commercially available, the internal standard with similar structures to these bile acids was used.

\section{Liquid chromatography and mass spectrometry}

The UPLC-MS/MS system was made up of an Agilent 1290 UPLC coupled to an Agilent 6470 triple quadrupole mass spectrometer equipped with an Agilent Jet Stream electrospray ionization (ESI) source (Agilent Technologies, Inc. Santa Clara, CA, USA). Samples $(1 \mu \mathrm{L})$ were separated on ZORBAX Eclipse Plus C18 column 
$\left(2.1 \times 100 \mathrm{~mm}, 1.8 \mu \mathrm{m}\right.$, Agilent, USA) at $45^{\circ} \mathrm{C}$. Mobile phases consisted of A (water with $0.005 \% \mathrm{HCOOH}, \mathrm{v} / \mathrm{v}$ ) and $\mathrm{B}$ (acetonitrile with $0.005 \% \mathrm{HCOOH}, \mathrm{v} / \mathrm{v}$ ). The elution gradient was set stepwise as follows: $1.23 \% \mathrm{~B}$ to $33 \% \mathrm{~B}$ for $2 \mathrm{~min} ; 2.33 \% \mathrm{~B}$ to $34 \%$

B for $4 \mathrm{~min}$; $3.34 \% \mathrm{~B}$ to $70 \% \mathrm{~B}$ for $5 \mathrm{~min}$. The flow rate was $0.6 \mathrm{~mL} / \mathrm{min}$. MS detection of bile acids were conducted in negative mode. Fragmentor and product ions for every bile acid were optimized through direct infusion of available bile acid standards to improve detective sensitivity. Multiple reaction monitoring (MRM) has been used for quantification of screening fragment ions.

\section{Data preprocessing}

Peak determination and peak area integration was performed with MassHunter Workstation software (Agilent, Version B.08.00) while auto-integration was manually inspected and corrected if necessary. The obtained peak areas of targets were corrected by appropriate internal standards (IS) and calculated response ratios were used throughout the analysis.

Table 130 Bile acids and 7 deuterium-labeled internal standards

$\begin{array}{cl} & \text { Abbreviation } \\ 1 & \text { THCA } \\ 2 & \text { T- } \alpha \text {-MCA } \\ 3 & \text { T- } \beta \text {-MCA } \\ 4 & \text { TCDCA } \\ 5 & \text { TCA } \\ 6 & \text { TDCA } \\ 7 & \text { TUDCA } \\ 8 & \text { TLCA } \\ 9 & \text { GCA } \\ 10 & \text { GHDCA } \\ 11 & \text { GCDCA } \\ 12 & \text { GDCA } \\ 13 & \text { GUDCA } \\ 14 & \text { GLCA } \\ 15 & \text { GHCA } \\ 16 & \text { CA } \\ 17 & \text { HCA } \\ 18 & \alpha-M C A\end{array}$

\section{Common Name}

Taurohyocholic acid

Tauro-alpha-muricholic acid

Tauro-beta-muricholic acid

Taurochenodeoxycholic acid

Taurocholic acid

Taurodeoxycholic acid

Tauroursodeoxycholic acid

Taurolithocholic acid

Glycocholic acid

Glycohyodeoxycholic acid

Glycochenodeoxycholic acid

Glycodeoxycholic acid

Glycoursodeoxycholic acid

Glycolithocholic acid

Glycohyocholic acid

Cholic acid

Hyocholic acid

Alpha-muricholic acid 


$\begin{array}{lll}19 & \beta-M C A & \text { Beta-muricholic acid } \\ 20 & \text { ACA } & \text { Allocholic acid } \\ 21 & \text { CDCA } & \text { Chenodeoxycholic acid } \\ 22 & \text { DCA } & \text { Deoxycholic acid } \\ 23 & \text { iso-DCA } & \text { Isodeoxycholic acid } \\ 24 & \text { HDCA } & \text { Hyodeoxycholic acid } \\ 25 & \text { UDCA } & \text { Ursodeoxycholic acid } \\ 26 & \text { nutriCA } & \text { Nutriacholic acid } \\ 27 & \text { 12-ketoDCA } & \text { 12-Ketodeoxycholic acid } \\ 28 & \text { LCA } & \text { Lithocholic acid } \\ 29 & \text { iso-LCA } & \text { Isolithocholic acid } \\ 30 & \text { w-MCA } & \text { Omega-muricholic acid } \\ & \text { TCA-D4 } & \\ & \text { 9-HODE-d4 } & \\ & \text { 6-keto PGF1 } \alpha-d 4 & \\ & \text { PGE2-d4 } & \end{array}$

Table 2 Concentrations of 30 bile acids calibration curves

\begin{tabular}{|c|c|c|c|c|c|c|c|c|c|c|c|c|}
\hline $\mathrm{nM}$ & 1 & 2 & 3 & 4 & 5 & 6 & 7 & 8 & 9 & 10 & 11 & 12 \\
\hline THCA & 2603.73 & 1301.87 & 650.93 & 325.47 & 162.73 & 81.37 & 40.68 & 20.34 & 10.17 & 5.09 & 2.54 & 1.27 \\
\hline T- $\alpha-\mathrm{MCA}$ & 2975.69 & 1487.85 & 743.92 & 371.96 & 185.98 & 92.99 & 46.50 & 23.25 & 11.62 & 5.81 & 2.91 & 1.45 \\
\hline T- $\beta$-MCA & 1952.80 & 976.40 & 488.20 & 244.10 & 122.05 & 61.02 & 30.51 & 15.26 & 7.63 & 3.81 & 1.91 & 0.95 \\
\hline TCDCA & 3301.98 & 1650.99 & 825.50 & 412.75 & 206.37 & 103.19 & 51.59 & 25.80 & 12.90 & 6.45 & 3.22 & 1.61 \\
\hline TCA & 2617.80 & 1308.90 & 654.45 & 327.23 & 163.61 & 81.81 & 40.90 & 20.45 & 10.23 & 5.11 & 2.56 & 1.28 \\
\hline TDCA & 2801.68 & 1400.84 & 700.42 & 350.21 & 175.11 & 87.55 & 43.78 & 21.89 & 10.94 & 5.47 & 2.74 & 1.37 \\
\hline TUDCA & 4312.91 & 2156.45 & 1078.23 & 539.11 & 269.56 & 134.78 & 67.39 & 33.69 & 16.85 & 8.42 & 4.21 & 2.11 \\
\hline TLCA & 3101.03 & 1550.52 & 775.26 & 387.63 & 193.81 & 96.91 & 48.45 & 24.23 & 12.11 & 6.06 & 3.03 & 1.51 \\
\hline GCA & 3973.20 & 1986.60 & 993.30 & 496.65 & 248.32 & 124.16 & 62.08 & 31.04 & 15.52 & 7.76 & 3.88 & 1.94 \\
\hline GHDCA & 3710.69 & 1855.35 & 927.67 & 463.84 & 231.92 & 115.96 & 57.98 & 28.99 & 14.49 & 7.25 & 3.62 & 1.81 \\
\hline GCDCA & 2544.48 & 1272.24 & 636.12 & 318.06 & 159.03 & 79.51 & 39.76 & 19.88 & 9.94 & 4.97 & 2.48 & 1.24 \\
\hline GDCA & 4892.15 & 2446.08 & 1223.04 & 611.52 & 305.76 & 152.88 & 76.44 & 38.22 & 19.11 & 9.55 & 4.78 & 2.39 \\
\hline GUDCA & 3224.95 & 1612.47 & 806.24 & 403.12 & 201.56 & 100.78 & 50.39 & 25.19 & 12.60 & 6.30 & 3.15 & 1.57 \\
\hline GLCA & 2633.83 & 1316.92 & 658.46 & 329.23 & 164.61 & 82.31 & 41.15 & 20.58 & 10.29 & 5.14 & 2.57 & 1.29 \\
\hline GHCA & 3221.65 & 1610.82 & 805.41 & 402.71 & 201.35 & 100.68 & 50.34 & 25.17 & 12.58 & 6.29 & 3.15 & 1.57 \\
\hline $\mathrm{CA}$ & 5139.88 & 2569.94 & 1284.97 & 642.48 & 321.24 & 160.62 & 80.31 & 40.16 & 20.08 & 10.04 & 5.02 & 2.51 \\
\hline $\mathrm{HCA}$ & 3793.72 & 1896.86 & 948.43 & 474.21 & 237.11 & 118.55 & 59.28 & 29.64 & 14.82 & 7.41 & 3.70 & 1.85 \\
\hline$\alpha-\mathrm{MCA}$ & 3548.96 & 1774.48 & 887.24 & 443.62 & 221.81 & 110.91 & 55.45 & 27.73 & 13.86 & 6.93 & 3.47 & 1.73 \\
\hline$\beta-\mathrm{MCA}$ & 3793.72 & 1896.86 & 948.43 & 474.21 & 237.11 & 118.55 & 59.28 & 29.64 & 14.82 & 7.41 & 3.70 & 1.85 \\
\hline ACA & 3793.81 & 1896.91 & 948.45 & 474.23 & 237.11 & 118.56 & 59.28 & 29.64 & 14.82 & 7.41 & 3.70 & 1.85 \\
\hline CDCA & 4457.80 & 2228.90 & 1114.45 & 557.23 & 278.61 & 139.31 & 69.65 & 34.83 & 17.41 & 8.71 & 4.35 & 2.18 \\
\hline DCA & 5858.38 & 2929.19 & 1464.60 & 732.30 & 366.15 & 183.07 & 91.54 & 45.77 & 22.88 & 11.44 & 5.72 & 2.86 \\
\hline iso-DCA & 3311.26 & 1655.63 & 827.81 & 413.91 & 206.95 & 103.48 & 51.74 & 25.87 & 12.93 & 6.47 & 3.23 & 1.62 \\
\hline HDCA & 4584.82 & 2292.41 & 1146.20 & 573.10 & 286.55 & 143.28 & 71.64 & 35.82 & 17.91 & 8.95 & 4.48 & 2.24 \\
\hline
\end{tabular}




\begin{tabular}{|c|c|c|c|c|c|c|c|c|c|c|c|c|}
\hline UDCA & 5986.19 & 2993.10 & 1496.55 & 748.27 & 374.14 & 187.07 & 93.53 & 46.77 & 23.38 & 11.69 & 5.85 & 2.92 \\
\hline nutriCA & 4096.68 & 2048.34 & 1024.17 & 512.09 & 256.04 & 128.02 & 64.01 & 32.01 & 16.00 & 8.00 & 4.00 & 2.00 \\
\hline 12-ketoDCA & 3328.55 & 1664.28 & 832.14 & 416.07 & 208.03 & 104.02 & 52.01 & 26.00 & 13.00 & 6.50 & 3.25 & 1.63 \\
\hline LCA & 4647.21 & 2323.61 & 1161.80 & 580.90 & 290.45 & 145.23 & 72.61 & 36.31 & 18.15 & 9.08 & 4.54 & 2.27 \\
\hline iso-LCA & 3451.94 & 1725.97 & 862.98 & 431.49 & 215.75 & 107.87 & 53.94 & 26.97 & 13.48 & 6.74 & 3.37 & 1.69 \\
\hline$\omega-\mathrm{MCA}$ & 6118.90 & 3059.45 & 1529.73 & 764.86 & 382.43 & 191.22 & 95.61 & 47.80 & 23.90 & 11.95 & 5.98 & 2.99 \\
\hline
\end{tabular}

\section{References}

[1] Lin, H., An Y. P., Tang H. R. and Wang Y. L. Alterations of Bile Acids and Gut Microbiota in Obesity Induced by High Fat Diet in Rat Model[J]. J Agric .Food Chem. 2019, 67(13): 3624-3632.

[2] Hofmann, A. F., L. R. Hagey and M. D. Krasowski . Bile salts of vertebrates: structural variation and possible evolutionary significance[J]. J Lipid Res. 2010, 51(2): 226-246.

[3] Kakiyama, G., A. Muto, H. Takei, H. Nittono, T. Murai, T. Kurosawa, A. F. Hofmann, W. M. Pandak and J. S. Bajaj . A simple and accurate HPLC method for fecal bile acid profile in healthy and cirrhotic subjects: validation by GC-MS and LC-MS[J]. J Lipid Res. 2014, 55(5): 978-990.

[4] Penno, C. A., D. Arsenijevic, T. Da Cunha, G. A. Kullak-Ublick, J.-P. Montani and A. Odermatt. Quantification of multiple bile acids in uninephrectomized rats using ultra-performance liquid chromatography-tandem mass spectrometry[J]. Anal methods. 2013, 5(5): 1155-1164.

[5] Thomas, C., R. Pellicciari, M. Pruzanski, J. Auwerx and K. Schoonjans. Targeting bile-acid signalling for metabolic diseases[J]. Nat Rev Drug Dis. 2008,7(8): 678-693. 